\title{
Exposure schedule for multiplexing holograms in photopolymer films
}

\author{
Allen Pu, MEMBER SPIE \\ Kevin Curtis, ${ }^{*}$ MEMBER SPIE \\ Demetri Psaltis, MEMBER SPIE \\ California Institute of Technology \\ 136-93 Caltech \\ Pasadena, California 91125 \\ E-mail: allenpu@sunoptics.caltech.edu
}

\begin{abstract}
An iterative method is introduced for determining the exposure schedule for multiplexing holograms in saturable recording materials, such as photopolymers. This method is designed to share all or part of the available dynamic range of the recording material among the holograms to be multiplexed. Using exposure schedules derived from this method, the authors find that the diffraction efficiency of DuPont's HRF15038 - and $100-\mu \mathrm{m}$ photopolymer scale is $(2.2 / \mathrm{M})^{2}$ and $(6.5 / \mathrm{M})^{2}$ respectively, where $M$ is the number of holograms recorded. Finally, 1000 holograms were multiplexed at a single location in the $100-\mu \mathrm{m}$ thick photopolymer using an exposure schedule derived with this method. () 1996 Society of Photo-Optical Instrumentation Engineers.
\end{abstract}

Subject terms: holographic data storage; exposure schedule; photopolymer; M-number.

Paper 31125 received Dec. 21, 1995; accepted for publication Mar. 19, 1996.

\section{Introduction}

Peristrophic multiplexing ${ }^{1}$ makes it possible to multiplex many holograms in thin films. As the number of holograms stored in these films increases, the optimal utilization of the available dynamic range becomes particularly important (since the diffraction efficiency scales as $1 / M^{2}$, where $M$ is the number of holograms multiplexed ${ }^{2}$ ). In this paper we present a method for recording equal strength holograms that utilizes all of the available dynamic range of the recording material. This method can also be generalized to record unequal strength holograms or to use only a portion of the available dynamic range. Furthermore, no assumptions about the physics of the recording material are made and the method takes into account all experimental parameters, including incident angles, incident intensities, and any special behavior of the recording material. We first demonstrate this method by recording 50 equal diffraction efficiency plane wave holograms using DuPont's HRF-150 photopolymer. Then, by recording different numbers of holograms, we measured the dependence of the diffraction efficiency on the number of holograms stored for the 38and $100-\mu \mathrm{m}$ thick photopolymers. Finally, 1000 holograms of a single random bit pattern were multiplexed using a recording schedule and the average diffraction efficiency was compared with the theoretical prediction.

\section{Exposure Schedule}

Figure 1 shows a plane wave hologram setup using peristrophic multiplexing. The signal and reference plane waves both had an incident angle of $30 \mathrm{deg}$ (outside) with respect to the normal on the photopolymer surface. The intensity of each beam was $250 \mu \mathrm{W} / \mathrm{cm}^{2}$ and the wavelength was $488 \mathrm{~nm}$. Shutters were used to block the beams

*Current affiliation: AT\&T Bell Labs, Murray Hill, New Jersey 07974. in-between hologram recording and to block the signal beam during reconstruction. The photopolymer was mounted on a rotational stage for peristrophic multiplexing. After each exposure, the recording material was rotated in plane by the rotational stage. This rotation causes the reconstruction from the stored hologram to come out in a different direction, allowing for another hologram to be stored at the same location. ${ }^{1}$ Reconstructed holograms are Fourier transformed by a lens and then filtered by a small aperture placed at the Fourier plane. This spatial filter allows one reconstruction to pass through while blocking all the rest. Different holograms can be read out by rotating the recording material so that its reconstruction is aligned with the spatial filter. The diffracted power of the reconstructed hologram is measured by a detector placed after the spatial filter.

The diffraction efficiency of 90 peristrophically (2-deg rotation between holograms) multiplexed plane wave holograms recorded with a uniform exposure schedule is shown in Fig. 2. The holograms were recorded in the HRF-150 $38-\mu \mathrm{m}$ thick photopolymer with $1 \mathrm{~mJ} / \mathrm{cm}^{2}$ incident exposure energy per hologram (2-s exposure at $0.5 \mathrm{~mW} / \mathrm{cm}^{2}$ total incident intensity). This photopolymer is fairly insensitive until it has been exposed to about $6 \mathrm{~mJ} / \mathrm{cm}^{2}$ of incident energy. The film then exhibits a quasi-linear recording behavior until the material saturates. Therefore, recording with a constant exposure schedule will result in nonuniform diffraction efficiency holograms. To overcome these problems, the photopolymer was preexposed to $6 \mathrm{~mJ} / \mathrm{cm}^{2}$ to bring it to the quasi-linear region by exposing the film to only the reference beam before any holograms were recorded. Then a recording schedule that compensated for the loss in grating strength as the material saturated was used to equalize the holograms.

The recording behavior of the photopolymer can be best characterized by the cumulative grating strength as a function of exposure energy. The curves shown in Fig. 3 were 


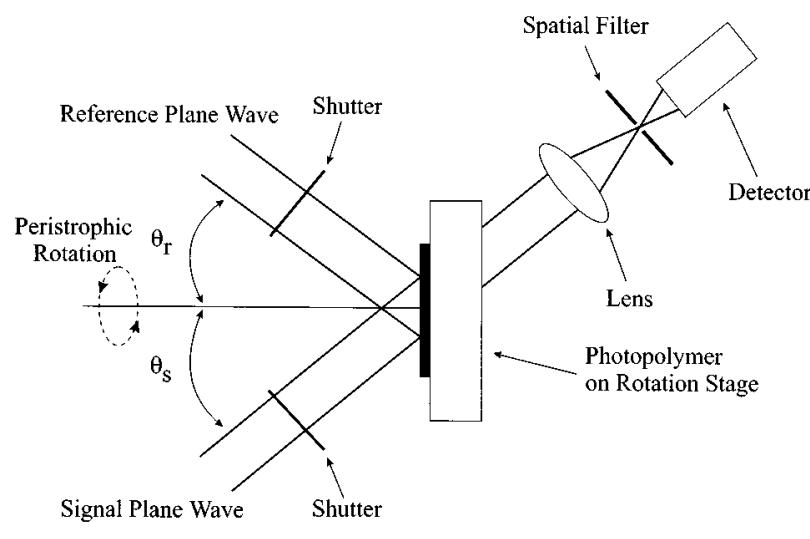

Fig. 1 A schematic diagram of the setup used to determine the recording schedule for 50 plane wave holograms.

obtained by integrating the square root of the diffracted power from the peristrophically multiplexed holograms recorded with $1,1.5$, and $2 \mathrm{~mJ} / \mathrm{cm}^{2}$ constant exposure schedules and $6 \mathrm{~mJ} / \mathrm{cm}^{2}$ preexposure. In Fig. 3, the origin of the exposure energy axis was reset to leave out the preexposure energy. From the figure, it can be seen that the cumulative grating strength grows quasi-linearly with exposure energy and then saturates. The final saturation grating amplitude for $1,1.5$, and $2 \mathrm{~mJ} / \mathrm{cm}^{2}$ exposure per hologram is different due to the "run-time" effect. ${ }^{3}$ For the three different exposure energies per hologram, the total exposure energy and incident intensities were kept constant at $90 \mathrm{~mJ} / \mathrm{cm}^{2}$ and $250 \mu \mathrm{W} / \mathrm{cm}^{2}$ per beam, respectively, while the number of holograms and exposure time for each of the three experiments were changed. After a hologram was written, the beams were blocked, the film rotated for peristrophic multiplexing, and $1.5 \mathrm{~s}$ allowed to elapse before a new hologram was recorded so that the table could settle. During that $1.5 \mathrm{~s}$, the material is losing dynamic range because once the photo-induced reaction is started, it continues even in the absence of light. This mechanism is the origin of the "run-time" effect. The $2 \mathrm{~mJ} / \mathrm{cm}^{2}$ per hologram curve wastes less dynamic range on self-enhancement since a larger fraction of the run time is spent on recording (4-s exposure per hologram for only 45 holograms). Therefore, the $2 \mathrm{~mJ} / \mathrm{cm}^{2}$ per hologram curve has the highest saturation grating amplitude.

The $1.5 \mathrm{~mJ} / \mathrm{cm}^{2}$ curve in Fig. 3 shows only a slight increase in saturation grating amplitude over the $1 \mathrm{~mJ} / \mathrm{cm}^{2}$ curve while the $2 \mathrm{~mJ} / \mathrm{cm}^{2}$ curve shows a more significant improvement. Theoretically, it should be the other way around since the reduction in total waiting time is greater going from 1 to $1.5 \mathrm{~mJ} / \mathrm{cm}^{2}$ per hologram than from 1.5 to $2 \mathrm{~mJ} / \mathrm{cm}^{2}$ per hologram. However, for the $1 \mathrm{~mJ} / \mathrm{cm}^{2}$ curve, 90 holograms were recorded and their diffraction efficiencies integrated. Therefore, the background noise from scattering gets integrated 90 times for the $1 \mathrm{~mJ} / \mathrm{cm}^{2}$ curve compared with only 45 times for the $2 \mathrm{~mJ} / \mathrm{cm}^{2}$ curve. This boosts the $1 \mathrm{~mJ} / \mathrm{cm}^{2}$ curve artificially closer to the $1.5-\mathrm{mJ} / \mathrm{cm}^{2}$ curve.

In order to derive an exposure schedule that equalizes the recorded holograms, we need to fit the recording curve

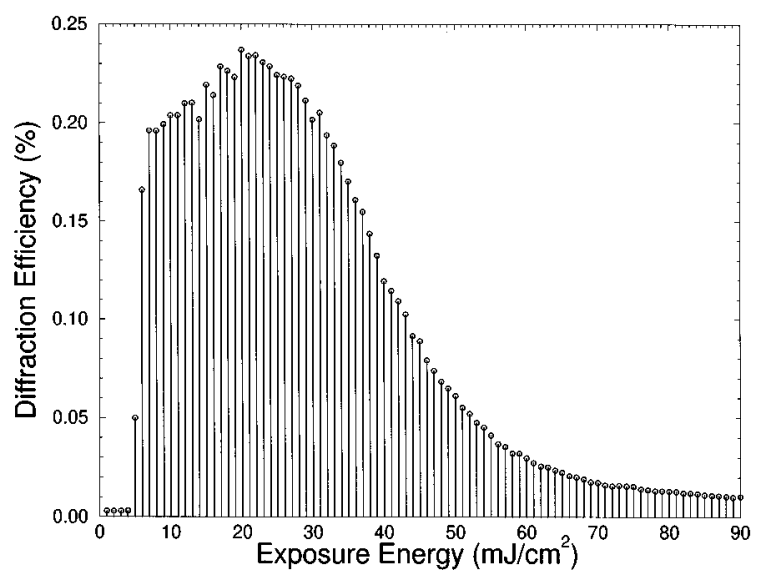

Fig. 2 The diffraction efficiency of 90 plane wave holograms recorded with a constant $1 \mathrm{~mJ} / \mathrm{cm}^{2}$ exposure per hologram.

of Fig. 3 to an analytical expression. The $1-\mathrm{mJ} / \mathrm{cm}^{2}$ per hologram curve was fitted to a sixth-order polynomial of the following form:

$A=a_{0}+a_{1} E+a_{2} E^{2}+a_{3} E^{3}+a_{4} E^{4}+a_{5} E^{5}+a_{6} E^{6}$,

where $A$ is the cumulative grating strength and $E$ is the cumulative exposure energy. A sixth-order polynomial was used because it was found to have sufficient degrees of freedom to fit closely with Fig. 3. By taking the derivative of Eq. (1), the grating strength growth rate as a function of exposure energy is obtained. Therefore, it is now straightforward to predetermine the strength of each hologram by appropriating the exposure energy correctly. For example, equal strength holograms can be achieved by allocating the entire dynamic range of the photopolymer equally among the holograms. Therefore, the desired exposure schedule becomes:

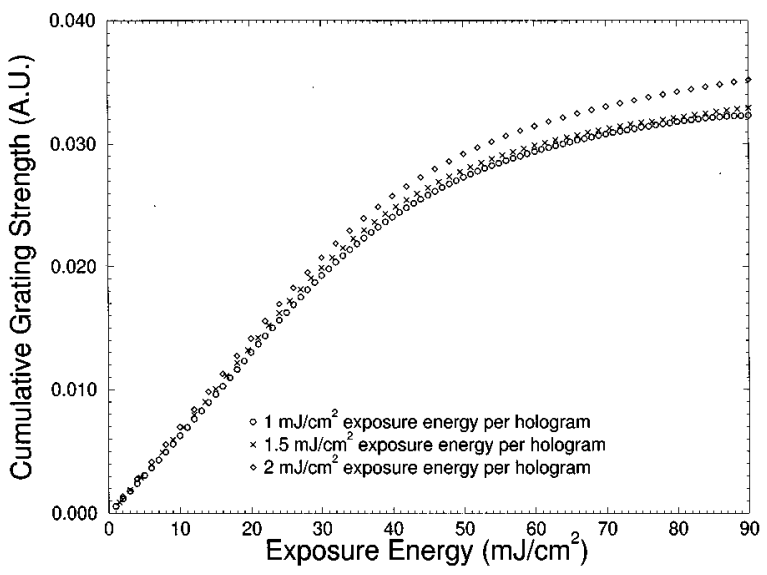

Fig. 3 The cumulative grating strength as a function of exposure energy curve for three different exposure energies per hologram. 


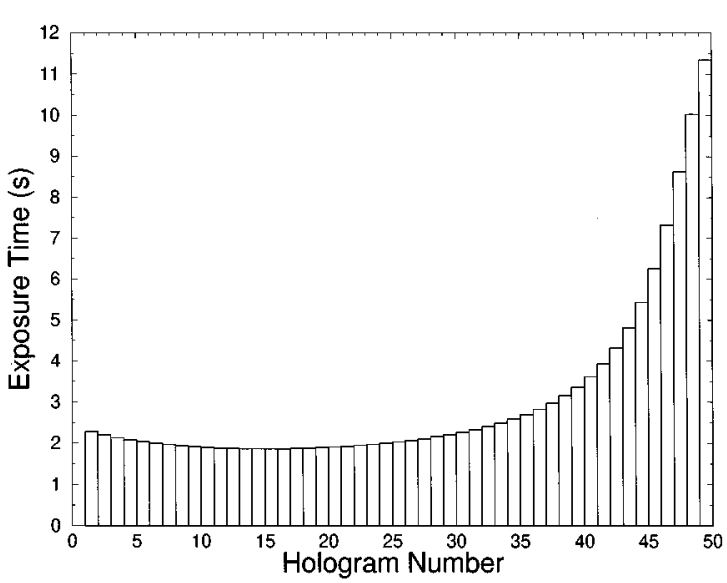

Fig. 4 A first iteration exposure schedule for 50 holograms.

$\frac{A_{\mathrm{sat}}}{M}=\left.\frac{\partial A}{\partial E}\right|_{E=\sum_{i=1}^{n-1} E_{i}} \times E_{n}$,

where $A_{\text {sat }}$ is the saturation grating strength from Fig. 3, $M$ is the number of stored holograms, $E_{i}$ is the amount of energy the photopolymer received in order to record the $i$ 'th hologram, and $E_{n}$ is the amount of energy required to record the $n$ 'th hologram. Each hologram is allocated $1 / M$ 'th of the photopolymer's dynamic range.

Using the same exposure intensity of $250 \mu \mathrm{W} / \mathrm{cm}^{2}$ per beam (so that our polynomial fit remains valid), the exposure schedule in terms of exposure time becomes:

$$
\begin{aligned}
t_{n}= & A_{\mathrm{sat}} / M\left[a_{1}+2 a_{2} \sum_{i=1}^{n-1} E_{i}+3 a_{3}\left(\sum_{i=1}^{n-1} E_{i}\right)^{2}\right. \\
& \left.+4 a_{4}\left(\sum_{i=1}^{n-1} E_{i}\right)^{3}+5 a_{5}\left(\sum_{i=1}^{n-1} E_{i}\right)^{4}+6 a_{6}\left(\sum_{i=1}^{n-1} E_{i}\right)^{5}\right] I,
\end{aligned}
$$

where $t_{n}$ is the exposure time of the $n$ 'th hologram and $I$ is the total incident intensity.

An exposure schedule for 50 holograms was calculated by the finite-step method (step size $=0.001 \mathrm{~mJ} / \mathrm{cm}^{2}$ ) using Eq. (3) and the parameters $a_{1}$ through $a_{6}$ obtained from fitting Fig. 3. Figure 4 shows the resulting exposure schedule. Notice that the exposure time per hologram increases as the film becomes saturated. The diffraction efficiencies of the 50 holograms that were recorded with this schedule are shown in Fig. 5. The grating strength of these 50 holograms is more uniformly distributed than the ones in Fig. 2 but is still not equal. This first iteration does not give us perfectly uniform holograms because some of the recording parameters have been changed. The exposure time per hologram is no longer a constant and we are now recording 50 holograms instead of the 90 used to generate the recording curve. Therefore these effects have to be remodeled. This

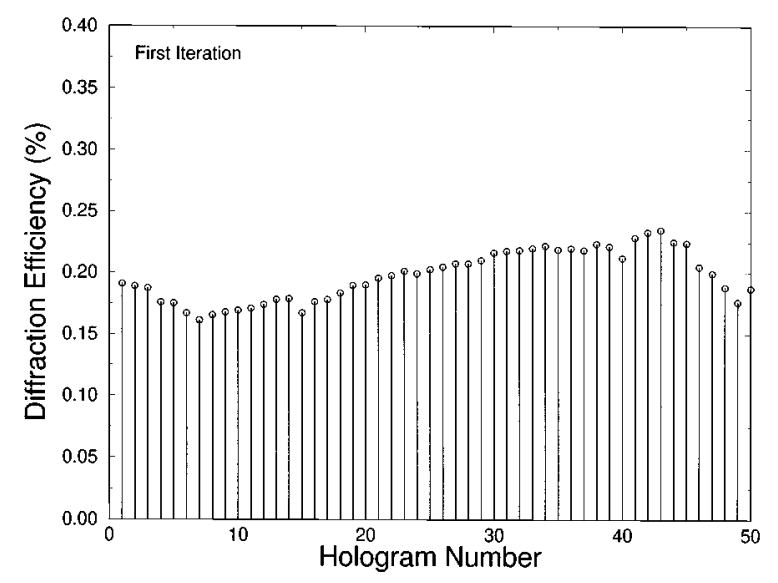

Fig. 5 The diffraction efficiency as a function of hologram number for holograms recorded with the first iteration exposure schedule.

can be done by iterating the previous procedure. Specifically, we integrate the square root of the diffracted power from the 50 holograms recorded with the schedule and plot it as a function of recording energy. We fit this curve to a sixth-order polynomial and then generate a new schedule using Eq. (3). Figure 6 shows the resulting cumulative grating strength as a function of exposure energy for the scheduled recording. Notice that the saturation grating strength for the scheduled recording is higher than the constant time recording. This is because the scheduled recording uses the dynamic range of the recording material more efficiently though better-managed exposures. When the photopolymer is sensitive, only a short exposure time is required. When the photopolymer is less sensitive, a longer exposure time activates the remaining monomers to achieve the same grating strength.

Figure 7 shows the diffraction efficiency of the 50 holograms that were recorded with the second iteration schedule generated from Fig. 6. From Fig. 7 it can be seen that the grating strength is quite uniform among the 50 holograms

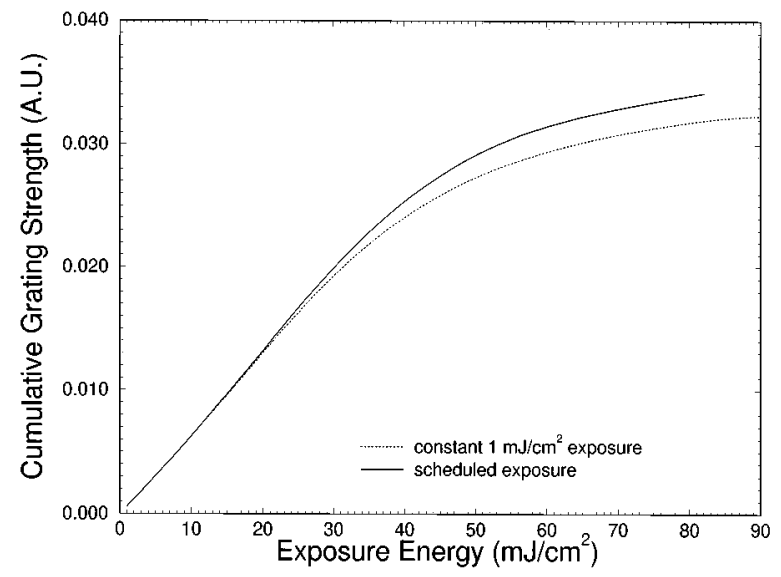

Fig. 6 A comparison between the cumulative grating strength of holograms recorded with a schedule and the $1 \mathrm{~mJ} / \mathrm{cm}^{2}$ constant exposure. 


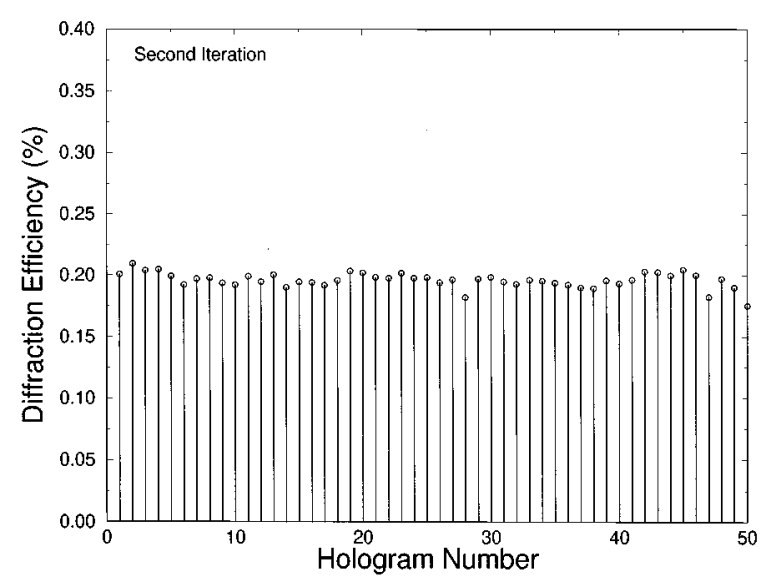

Fig. 7 The diffraction efficiency as a function of hologram number for holograms recorded with the second iteration exposure schedule.

and the standard deviation is only 0.006 (in percent diffraction efficiency). The above procedure can be repeated until the desired uniformity is reached.

\section{Diffraction Efficiency as a Function of Number of Holograms}

This simple method of determining the recording schedule can be used for any number of holograms and any recording setup. Moreover, since we did not make use of the physical properties of the recording medium in deriving the exposure schedule, this method for recording equalized holograms can be applied to any saturable recording medium. We can use this recording schedule to determine how the diffraction efficiency per hologram decreases as the number of multiplexed holograms increases. Previously, in the determination of the exposure schedule for equal-strength holograms, each hologram was allocated a grating strength of $A_{\text {sat }} / M$. Since diffraction efficiency is proportional to the grating strength squared, the diffraction efficiency scales as $\alpha / M^{2}$, where $\alpha$ is the proportionality constant. To determine $\alpha, 10,25,50,75$, and 90 peristrophic plane wave holograms were recorded using schedules obtained with the above method for the HRF-150-38 and HRF-150-100 photopolymer films (for the 100- $\mu$ m thick film, only $25,50,75$, and 90 plane wave holograms were recorded). The holograms were all equal strength and used the entire dynamic range of the recording material. The resulting diffraction efficiencies for these cases are plotted in Fig. 8. From fitting Fig. 8, the square root of $\alpha$ is determined to be 2.2 for the $38-\mu \mathrm{m}$ thick photopolymer and 6.5 for the $100-\mu \mathrm{m}$ thick photopolymer. For photorefractives, the square root of $\alpha$ is called the $M / \#,{ }^{2}$ and we also call the square root of $\alpha$ the $M / \#$ for the photopolymer.

The $M / \#$ of a recording material varies, depending on experimental conditions such as material thickness, absorption, recording geometry, and the ratio of reference to signal beam intensity. After the $M / \#$ of a material is measured for a particular setup, it can be used to predict the diffrac-

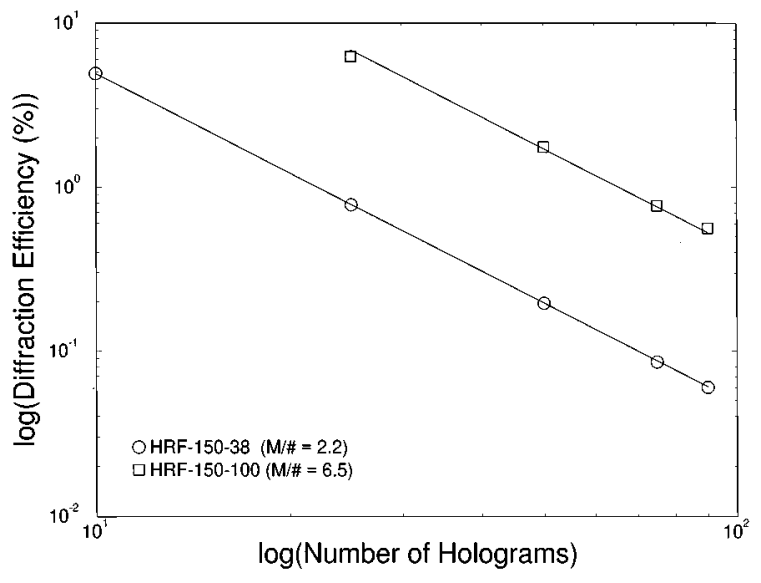

Fig. 8 The diffraction efficiency as a function of number of holograms multiplexed at the same location.

tion efficiency obtainable for the number of holograms to be multiplexed. For example, if we were to multiplex 1000 holograms in the HRF-150-100 photopolymer, the theory predicts a diffraction efficiency of $(6.5 / 1000)^{2} \simeq 4 \times 10^{-5}$ per hologram. We set up an experiment to verify the theory by storing 1000 image plane holograms of a random 100 $\times 100$ pixel pattern. The size of each pixel was $100 \times 100$ $\mu \mathrm{m}$ and it was made from a photographic glass plate. To multiplex 1000 holograms in the $100-\mu \mathrm{m}$ thick photopolymer, peristrophic and angle multiplexing were combined. Figure 9 shows the setup. The reference and signal beams were initially incident at $\pm 30 \mathrm{deg}$ from the film's normal. Angle multiplexing was achieved by rotating the recording material. The reference and signal beam intensities were 1 $\mathrm{mW} / \mathrm{cm}^{2}$ per beam. A thousand holograms were multiplexed at the same location by recording 100 peristrophic holograms at each of 10 angle multiplexing positions. The film was rotated in plane by $1.8 \mathrm{deg}$ to realize peristrophic multiplexing. Angular positions were separated by $1.5 \mathrm{deg}$. There was a 1-s delay between holograms to allow the rotation stages to settle completely. A diffraction efficiency of $\sim 4 \times 10^{-5}$ (corrected for the half-on, half-off nature of

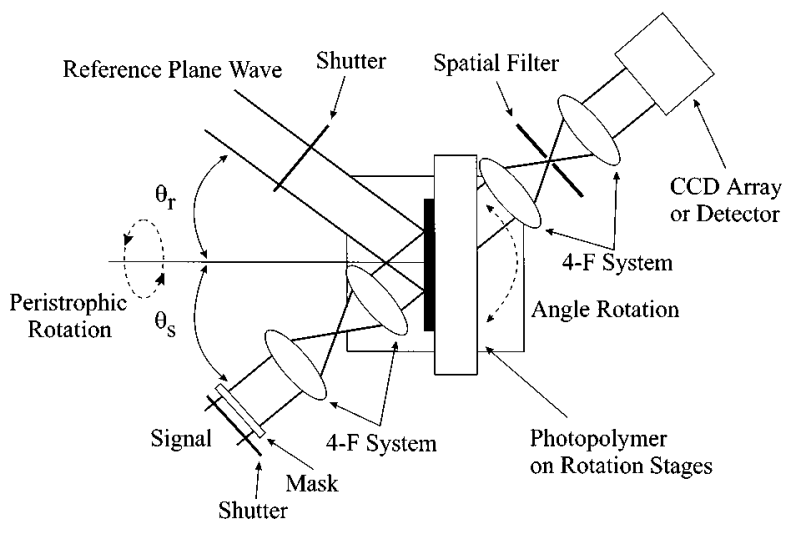

Fig. 9 A schematic diagram of the setup used to record 1000 holograms. 


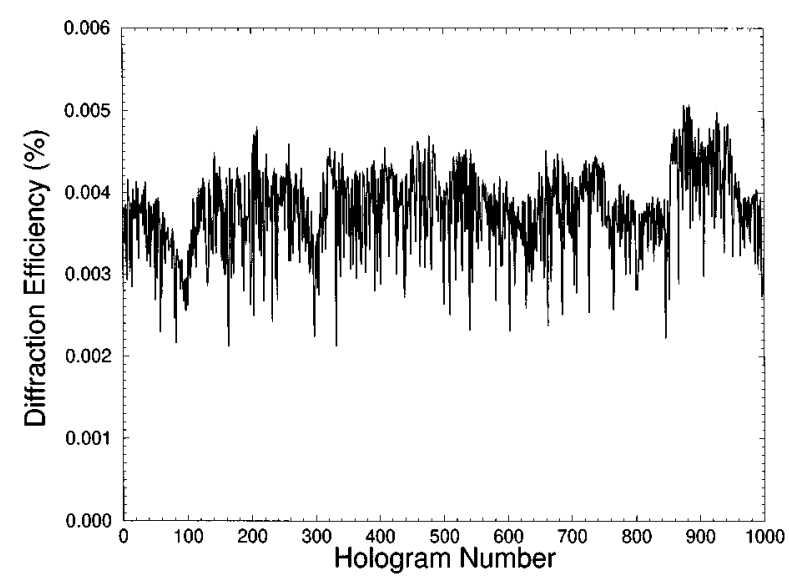

Fig. 10 The diffraction efficiency as a function of hologram number for the 1000-hologram experiment.

the binary amplitude mask) was obtained using six iterations of the recording schedule. The measured diffraction efficiency matches very well with theory. Figure 10 shows the resulting diffraction efficiency of the 1000 holograms. The sharp nonlinearity in the curve is due to stage instability during recording. The rotation stages used for peristrophic and angle multiplexing are servo-units and they sometimes twitch unpredictably. Other causes for the nonuniformity are photopolymer film shrinkage during recording and laser fluctuations. Shrinkage causes the holograms to Bragg match at a slightly different reference beam angle than that used to record the hologram. Therefore, to measure the true diffraction efficiency of the holograms, it is necessary to find the maximum diffracted power by changing the reference beam angle for each hologram. One way to achieve this is to scan the reference beam angle in small steps during reconstruction and note the maximum diffracted power for each hologram. However, this would require us to take power measurements at an order of magnitude higher than 1000 points. At $4 \mathrm{~s}$ per point (to allow the power meter to settle), the entire measurement could run into days. Therefore, we opted to measure the diffracted power at the same reference beam angle as that used for recording. The shrinkage effect was not noticeable in the other experiments discussed previously because the signal and the reference beams were both fixed at $30 \mathrm{deg}$ with respect to the surface normal and no angle multiplexed holograms were recorded (for symmetric recording, $\theta_{s}=\theta_{r}$, shrinkage does not change the Bragg condition).

The signal-to-noise ratio of the original image and the reconstructions can be calculated using ${ }^{4}$ :

$S N R=\frac{m_{2}-m_{1}}{\sqrt{\sigma_{1}^{2}+\sigma_{2}^{2}}}$

where $m_{1}$ and $\sigma_{1}$ are the mean and standard deviations for the dark areas. Similarly, $m_{2}$ and $\sigma_{2}$ are the mean and standard deviations for the white areas. The average SNR for the 1000 reconstructions is approximately 7.5 while the

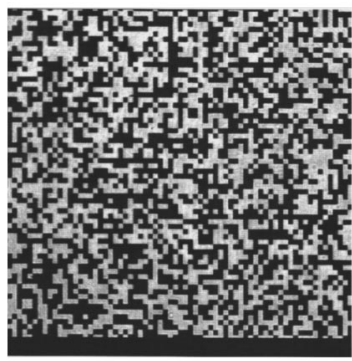

Original Image

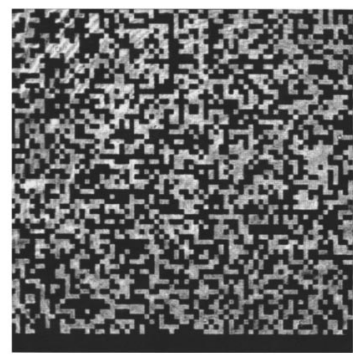

Reconstruction
Fig. 11 The image of the random data mask used to record 1000 holograms and one of the reconstructions.

SNR of the original image is 12 (Fig. 11). Assuming Gaussian noise statistics, this gives a bit error rate of better than $10^{-12}$ for the reconstructions.

\section{Conclusion}

We have presented a method for determining a recording schedule for photopolymer films that uses all the available dynamic range and takes into account the setup and runtime considerations. This method can be use to record equal-strength holograms in any saturable material. Using the schedule, we determined that the diffraction efficiency scales are $(2.2 / M)^{2}$ and $(6.5 / M)^{2}$ for DuPont's HRF-150-38 and -100 photopolymers, respectively, where $M$ is the number of holograms stored. This means that about 2000 holograms can be multiplexed in HRF-150-38 for a diffraction efficiency of $\sim 10^{-6}$ per hologram. Similarly, for the HRF-150-100 film, 6500 holograms can be multiplexed at a single location for the same diffraction efficiency. Furthermore, 1000 holograms of a random bit pattern were recorded using the schedule process, and the resulting average diffraction efficiency matches very well with the $M / \#$ prediction.

\section{Acknowledgments}

We would like to thank Geoffrey Burr for all his helpful comments. We gratefully acknowledge the support of the Air Force Office of Science Research and the National Science Foundation Engineering Research Center, Center for Neuromorphic Systems Engineering for this work.

\section{References}

1. Kevin Curtis, Allen Pu, and Demetri Psaltis, "A method for holographic storage using peristrophic multiplexing," Opt. Lett. 19(13), 993-994 (1994).

2. Fai Mok, Geoffrey Burr, and Demetri Psaltis, "A system metric for holographic memory systems," Opt. Lett. 21(12), 886-898 (1996).

3. B. L. Booth, "Photopolymer material for holography," Appl. Opt. 14(3), 593-601 (1975).

4. Athanasios Papoulis, Probability, Random Variables, and Stochastic Processes, 3rd ed., McGraw-Hill, New York (1991). 
Pu, Curtis, and Psaltis: Exposure schedule for multiplexing holograms ...

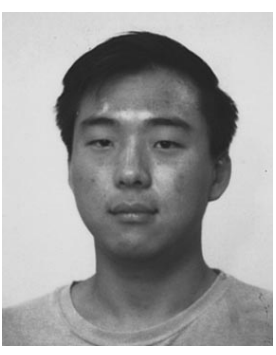

Allen Pu received his BS from The Cooper Union in 1992 and an MS from the California Institute of Technology, Pasadena, in 1993, both in electrical engineering. He is currently a PhD candidate at the California Institute of Technology, conducting research in optical computing with special interests in holographic data storage and optical correlators.

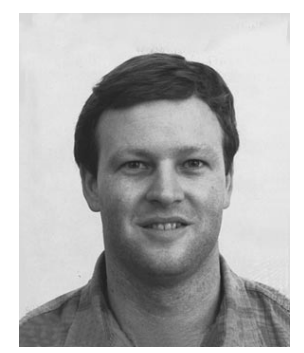

Kevin Curtis received his BS, MS, and $\mathrm{PhD}$ degrees in electrical engineering in 1990, 1992, and 1994, respectively, all from the California Institute of Technology, Pasadena. Dr. Curtis has authored or coauthored more than 20 publications on holography. $\mathrm{He}$ is a member of the Optical Society of America and SPIE, and his current research interests include holography, optical information processing, photopolymers, photorefractives, and optical memory. He is a member of the technical staff at AT\&T Bell Labs, Murray Hill, New Jersey.

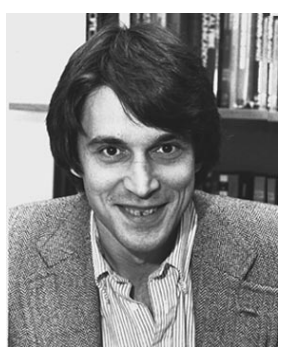

Demetri Psaltis received a BSc in electrical engineering and economics in 1974 and $\mathrm{MSc}$ and $\mathrm{PhD}$ degrees in electrical engineering in 1975 and 1977, respectively, all from Carnegie Mellon University, Pittsburgh. He remained at Carnegie Mellon, as a research associate and later as a visiting assistant professor, for 3 years. In 1980 , he joined the faculty at the California Institute of Technology, where he is now executive officer of the Computation and Neural Systems Department, professor of electrical engineering, and a consultant to industry. His research interests are in optical memories, optical information processing, holography, pattern recognition, neural networks, and optical devices. Dr. Psaltis has authored or co-authored more than 200 publications. He is a fellow of the Optical Society of America and received the International Commission on Optics Prize in 1989. 